\title{
Application of Laplace Transform for RLC Circuit
}

\author{
Mya Thida Hlaing \\ Department of Engineering \\ Mathematics \\ Technological University \\ Thanlyin, Myanmar
}

\author{
Wah Wah Aung \\ Department of Engineering \\ Mathematics \\ Technological University \\ Thanlyin, Myanmar
}

\author{
Thae Thae Htwe \\ Department of Engineering \\ Mathematics \\ Technological University \\ Pathein, Myanmar
}

\begin{abstract}
In this paper, Laplace transform is discussed and electric circuit problem as second order nonhomogeneous linear ordinary differential equation with constant coefficients is formulated. Then, this problem is solved by using Laplace transform method and analytical method. And then, ELC circuit acting over a time-interval will be solved by applying only Laplace transform method.
\end{abstract}

Keywords: Laplace transform. Ordinary differential equation. Electric circuit. Kirchhoff's Voltage Law. Time-shifting.

\section{INTRODUCTION}

The Laplace transform is an integral transform in mathematics. The transform has many applications in science and engineering such as first order ODE modeling (RL \& RC)circuits with no AC source and with a DC source, second order ODE modeling (series \& parallel RLC) circuits with no DC source and with AC source, and so on. Laplace transform of unit step function is suitable for solving ODEs with complicated right sides of considerable engineering interest such as single waves, inputs (driving forces) act for some time only. Laplace transforms are usually restricted to functions of $t$ with $t \geq 0$. Laplace transformation from the time domain to the frequency domain transforms second order ordinary differential equations into algebraic equations. The required solutions are obtained by applying definition and some properties of Laplace transform as follows.

\subsection{Definition of Laplace transform:}

Let $y(t)$ be a function of $t$ specified for $t>0$, then the integral $\int_{0}^{\infty} e^{-s t} y(t) d t$ is called Laplace transform of $y(t)$ and is denoted by $L\{y(t)\}$ or $Y(s)$.

$$
\text { i.e. } L\{y(t)\}=\int_{0}^{\infty} e^{-s t} y(t) d t=Y(s)
$$

\subsection{Properties of Laplace transform:}

Linearity property:

If $f(t)$ and $g(t)$ are any functions of $t$, a and $b$ are any constants, then

$$
L\{a f(t)+b g(t)\}=a L\{f(t)\}+b L\{g(t)\} .
$$

Laplace transform of derivatives:

If $L\{y(t)\}=Y(s)$, then

$L\left\{y^{[n]}(t)\right\}=s^{n} Y(s)-s^{n-1} y(0)-s^{n-2} y^{s}(0)-\cdots-y^{(n-1)}(0)$.

Inverse Laplace transform:

If $L\{y(t)\}=Y(s)$, then $y(t)=L^{-1}\{Y(s)\}$ is called inverse Laplace transform of $\mathrm{Y}(\mathrm{s})$.
S-shifting property:

If $L\{y(t)\}=Y(s)$, then $L\left\{e^{a t} y(t)\right\}=Y(s-a)$.

Laplace transform of unit step function:

The unit step function is a typical engineering function made to measure for engineering applications, which often involve functions are either "off" or "on".

$u(t-a)=\left\{\begin{array}{l}0 \text { if } t<a \\ 1 \text { if } t>a\end{array}\right.$

is also called Heaviside function.

T-shifting Property:

If $L\{y(t)\}=Y(s)$, then $L\{y(t-a) u(t-a)\}=Y(s) e^{-a s}$

which is often modeled in a RLC circuit by a voltage source in series with a switch.

\section{APPLICATION OF LAPLACE TRANSFORM ON ODES}

This section, the definition of ordinary differential equation and the application of Laplace transform on second order linear ODE are described.

An ordinary differential equation (ODE) is a differential equation containing one or more functions of one independent variable and the derivatives of those functions.

The Laplace transform is a useful method in solving linear ODE with constant coefficients.

Consider second order nonhomogeneous initial value problem

$y^{t s}+a y^{s}+b y=r(t), y\left(t_{0}\right)=k_{0} \cdot y^{t}\left(t_{0}\right)=k_{1}$

If Laplace transform on both sides of (1) is taken, the Laplace transform of derivatives and initial conditions are used, then algebraic equation is got. And then, the required solution is obtained by applying the inverse Laplace transform and sshifting property.

Example: $y^{t s}+4 y^{s}+20 y=120, y(0)=0, y^{t}(0)=0$.

Solution: Taking Laplace transform on both sides

$L\left[y^{\prime \prime}\right]+4 L\left[y^{\prime}\right]+20 L[y]=L[120]$ 
Using Laplace transform of derivatives and initial condition

$$
\begin{aligned}
& \left(s^{2}+4 s+20\right) Y=\frac{120}{s} \\
& \therefore(s)=\frac{120}{s\left(s^{2}+4 s+20\right)}
\end{aligned}
$$

By using partial fraction method, inverse Laplace transform, and s-shifting property

$y(t)=6 t-6 e^{-2 t} \cos 4 t-3 e^{-2 t} \sin 4 t$.

\section{MODELING RLC CIRCUIT}

This section, the definition of electric circuit, Kirchhoff's Voltage Law and modeling to RLC circuit according to KVL are presented.

An electric circuit is a path in which electrons from a voltage or current source flow. The point where those electrons enter an electric circuit is called the source of electrons highvoltage direct current transmission uses big converters.

Kirchhoff's Voltage Law states that the sum of all voltages around a closed loop in any circuit must be equal to zero. This is a consequence of charge conservation and also conservation of energy.

Consider the circuits are basic building blocks of such networks. They contain three kinds of components, a resistor of resistance $\mathrm{R} \Omega(\mathrm{ohms})$, an inductor of inductance $\mathrm{L}$ $\mathrm{H}$ (henrys) and a capacitor of capacitance $\mathrm{C} \mathrm{F}$ (farads) are wired in series circuit, the same current flows through all components of the circuit, and connected to a generator or an electromotive force $\mathrm{E}(\mathrm{t}) \mathrm{V}$ (volts), sinusoidal as in following figure

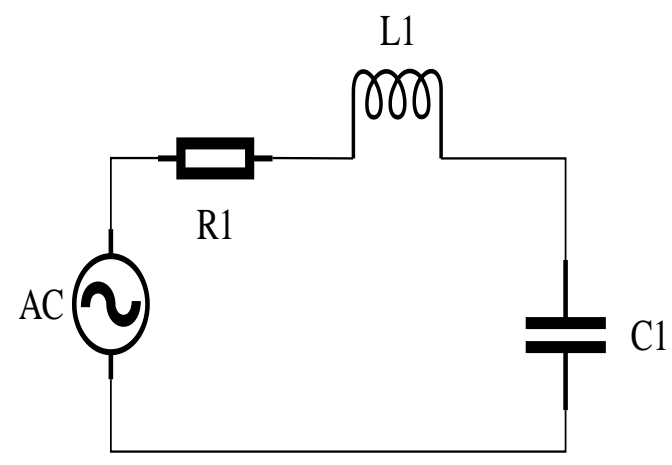

Figure1. RLC-circuit

The circuit is a closed loop, and the impressed voltage $E(t)$ equals the sum of the voltage drops across the three elements $\mathrm{R}, \mathrm{L}, \mathrm{C}$ of the loop.

According to Kirchhoff's Voltage Law, the above figure for an RLC-circuit with electromotive force

$E(t)=E_{0} \sin \omega t\left(E_{0}\right.$ constant $)$ as a model

$E_{L}+E_{R}+E_{C}=E(t)$

$L i^{t}+R i+\frac{1}{C} \int i d t=E(t)=E_{0} \sin \omega t$

or $q^{t s}+R q^{b}+\frac{1}{c} q=E_{0} \sin \omega t$

here $\mathrm{q}$ is the charge on the capacitor, $\mathrm{i}$ is the current in the circuit : $i(t)=\frac{d q}{d t}$ or $q(t)=\int i(t) d t$

and differentiate (3)

$L i^{s s}+R i^{t}+\frac{1}{c} i=E_{0} \cos \omega t$.

This equation is a modeling RLC circuit as a second-order non-homogeneous linear ODE with constant coefficients.

\section{APPLICATION TO RLC- CIRCUIT}

Many science and technical problems are built as mathematical model in various fields. These models are solved by applying kinds of mathematical methods. Among them, now present modeling RLC circuit as second order non homogeneous linear ODE with constant coefficients by applying the analytical method and Laplace transform method. And then, solve RLC circuit problem given time interval by applying Laplace transform of time shifting property.

\subsection{Analytical and Laplace transform methods application to RLC-circuit problem}

A circuit has in series an electromotive force of $600 \mathrm{~V}$, a resistor of $24 \Omega$, an inductor of $4 \mathrm{H}$, and a capacitor of $10^{-2}$ farads. If the initial current and the initial charge on the capacitor are both zero, Find the charge and the current at time $\mathrm{t}>0$.

According to Kirchhoff's Voltage Law

$$
\begin{aligned}
& E_{L}+E_{R}+E_{C}=E(t) \\
& L q^{r s}+R q^{s}+\frac{1}{c} q=600 \\
& q^{s t}+6 q^{s}+25 q=150
\end{aligned}
$$

is second-order non homogeneous linear ODE.

Applying Analytical method

The corresponding homogeneous linear ODE of (5) is

$q^{* s}+6 q^{p}+25 q=0$

the corresponding characteristic equation of (6) is

$\lambda^{2}+6 \lambda+25=0$ and $\lambda=-3 \pm 4 i$

the general solution of (6) is

$q_{h}(t)=e^{-a t}(A \cos 4 t+B \sin 4 t)$

using the method of undetermined coefficients, $q_{p}(t)=6$

then, the general solution of (5) is

$$
q(t)=e^{-3 t}(A \cos 4 t+B \sin 4 t)+6
$$

differentiating (7) and using initial condition, the charge:

$$
q(t)=6-6 e^{-3 t} \cos 4 t-4.5 e^{-3 t} \sin 4 t
$$

and the current: $i(t)=37.5 e^{-3 t} \sin 4 t$.

Applying Laplace transform method

Taking Laplace transform on both sides of (5)

$L\left\{q^{\prime \eta}\right\}+6 L\left\{q^{n}\right\}+25 L\{q\}=L\{150\}$

using Laplace transform of derivative and initial condition 


$$
Q(s)=\frac{150}{s\left[s^{2}+6 s+25\right]}
$$

by partial fraction method,

$Q(s)=\frac{6}{s}-\frac{6(s+3)+18}{(s+3)^{2}+4^{2}}$

applying inverse transform and s-shifting property,

the charge:

$$
q(t)=6-6 e^{-3 t} \cos 4 t-4.5 e^{-3 t} \sin 4 t
$$

and the current: $\quad i(t)=37.5 e^{-3 t} \sin 4 t$.

\subsection{Output of an RLC-circuit to a sinusoidal input acting over a time interval}

An inductance of 0.4 henry, a resistor of $12 \mathrm{ohms}$ and a capacitor of 0,0125 farad are connected in series with an electromotive force of $220 \sin 10 t$ volts. At $t=0$, the charge on the capacitor and current in the circuit is zero. Find the current where $\mathrm{E}(\mathrm{t})$ is sinusoidal, acting for a short time interval

$E(t)= \begin{cases}220 \sin 10 t & \text { if } 0<t<2 \pi \\ 0 & \text { if } t \geqslant 2 \pi\end{cases}$

Modeling by KVL,

$L i^{s s}+R i^{s}+\frac{1}{C} i=E^{s}, \quad i(0)=0, i^{t}(0)=0$

$i^{t s}+30 i^{t}+200 i=550 \times 10 \cos 10 t$

The above equation is second-order nonhomogeneous linear ODE.

Applying Laplace transform of derivative and time shifting property

$$
\begin{aligned}
& {\left[s^{2} I(s)-s i(0)-i^{t}(0)\right]+30[s I(s)-i(0)]+200 I(s)} \\
& =5500\left[\frac{s}{s^{2}+10^{2}}\left(1-e^{-2 \pi s}\right)\right]
\end{aligned}
$$

using initial conditions and partial fraction method,

$I(s)=\frac{22}{s+20}-\frac{27.5}{s+10}+\frac{5.5 s}{s^{2}+10^{2}}+\frac{165}{s^{2}+10^{2}}\left(1-e^{-2 \pi s}\right)$

Applying the inverse Laplace transform on both sides and tshifting property, the current is

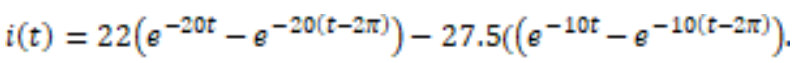

\section{CONCLUSIONS}

Through this paper, we present the application of Laplace transform and RLC-circuit is modeled as second order nonhomogeneous linear ODE. When RLC-circuit problem is solved by applying the two methods, the charge and the current of this problem are the same in subsection 4.1. But, RLC-circuit acting over time interval can be solved by applying only Laplace transform method in subsection 4.2. Therefore, linear ordinary differential equations with constant coefficients can be easily solved by the Laplace Transform method without finding the general solution, particular solution and the arbitrary constants as analytical method. Thus, Laplace transform method is more effective tool to solve complex problems than the analytical method in various fields.

\section{ACKNOWLEDGMENTS}

First, the authors would like to acknowledge the support of the papers for their references. The authors are deeply grateful to their adorable benefactor parents and teachers who gave their knowledge, useful discussions, powerful encouragement, invaluable suggestions and interest help through their life.

\section{REFERENCES}

[1] Laplace Transforms, "Murray. R. Spiegel," Higher Engineering Mathematics, Mc-Graw Hill Publication.

[2] Ordinary Differential Equations, "Shepley L.Ross," Third Edition ("John Wiley \& Sons," New York, 1980).

[3] Advanced Engineering Mathematics, "Erwin Kreysizig," $9^{\text {th }}$ Edition (John Wiley \& Sons, New York, 2006).

[4] Engineering Mathematics Volume II, "Madhumangal Pal and Anita Pal," 2011.

[5] Advanced Differential Equations, "Wilsky Erasmus," Inidia, 2014.

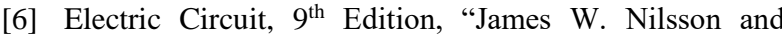
Susan A. Riedel." 